Genetic engineering

\title{
Friss oder stirb! Erweiterung des Substratspektrums von P. putida
}

HANNES LÖWE, ANDREAS KREMLING, KATHARINA PFLÜGER-GRAU

PROFESSUR FÜR SYSTEMBIOTECHNOLOGIE, FAKULTÄT FÜR MASCHINENWESEN, TU MÜNCHEN

The gram-negative bacterium Pseudomonas putida is of increasing interest for industrial applications due to its intrinsic resistance to a broad range of stresses, its metabolic versatility, and the availability of genetic tools. Our group aims to introduce new metabolic pathways by genetic engineering to further expand the metabolic spectrum of this microorganism. Here, we summarize the process of engineering a sucrose consuming strain of $P$. putida, the obstacles found on the way, and how they were overcome to achieve a stable phenotype.

DOI: $10.1007 / \mathrm{s} 12268-021-1568-6$

(C) Die Autoren 2021

Aufgrund seiner Robustheit, der Resistenz gegenüber einer Vielzahl von Stressfaktoren, seiner genetischen Zugänglichkeit und seines breiten Substratspektums, erfreut sich Pseudomonas putida zunehmenden Interesses für biotechnologische Anwendungen [1-4]. Die intrinsische Resistenz von
P. putida liegt u. a. in der einzigartigen metabolischen Architektur begründet, die es dem Organismus erlaubt die Menge der zur Verfügung stehenden Reduktionsäquivalente anzupassen [5, 6] sowie in der verhältnismäßig dichten äußeren Membran.
In biotechnologischen Anwendungen ist es von Interesse, die Produktionskosten so niedrig wie möglich zu halten, dazu gehört auch der Einsatz günstiger Kohlenstoffquellen. Eine Alternative zu der noch weitverbreiteten Glucose ist die Nutzung von Saccharose, einem Nebenprodukt der Zuckerherstellung aus Zuckerrüben, Zuckerrohr oder Zuckerpalmen. Ein Schwerpunkt unserer Arbeit ist die Erweiterung des Substratspektrums von P. putida, sodass auch bis dato nicht nutzbare Stoffe als C-Quelle zur Verfügung stehen. In diesem Artikel möchten wir kurz den Weg zur Entstehung eines Saccharose-verstoffwechselnden Stamms durch rationales genetisches Design nachzeichnen.

Eingeschränktes Wachstum auf Saccharose in $P$. putida durch $\csc A B$ aus $E$. coli W

Um P. putida das Wachstum auf Saccharose zu ermöglichen, ist es nötig, die Aufnahme und Spaltung der Saccharose zu gewährleisten. Dazu setzten wir die Gene $\csc A$ und
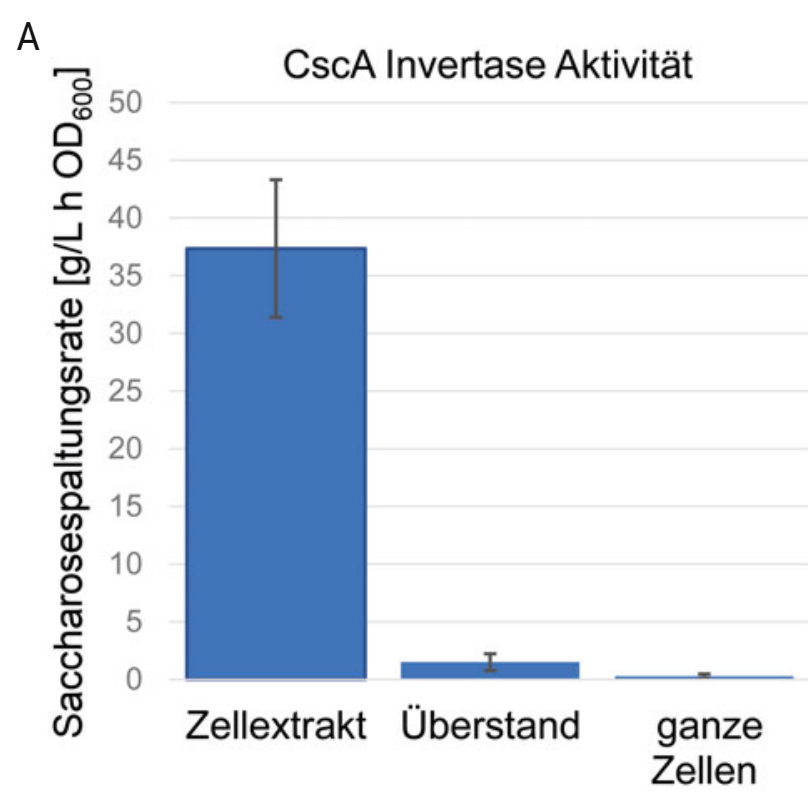

B
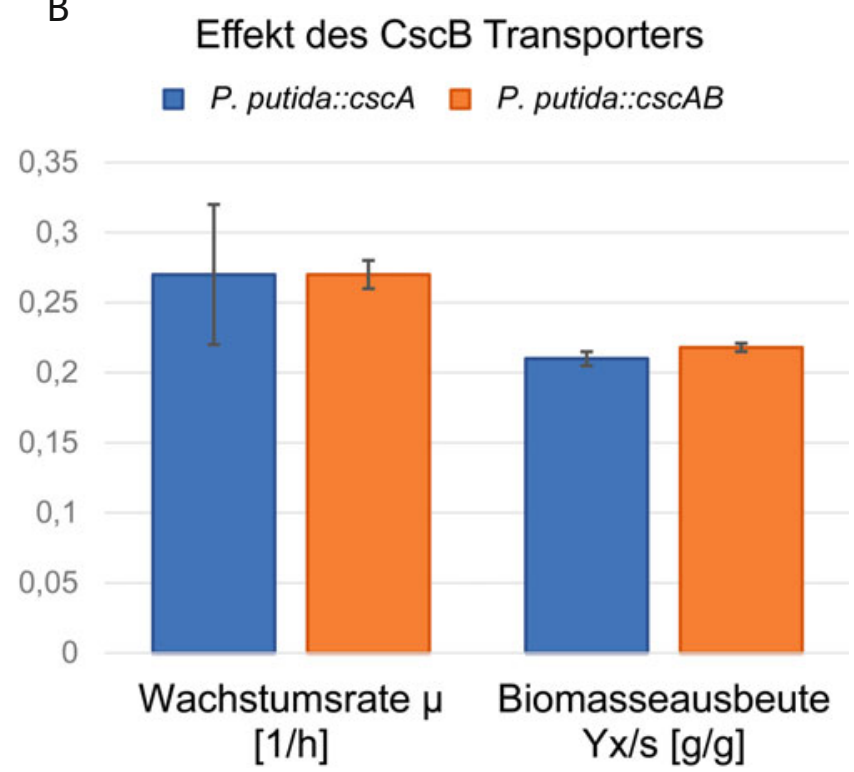

$\Delta$ Abb. 1: Funktionalität der Gene $\csc A B$ aus Escherichia coli W in Pseudomonas putida. A, Aktivität der Invertase CscA in Zellextrakt, Überstand und ganzen Zellen von $P$. putida cscAB. Der Großteil der Aktivität ist im intrazellulären Bereich nachzuweisen. B, Wachstumsraten und Biomasseausbeute von $P$. putida $\csc A$ und $P$. putida $\csc A B$ (Mittelwerte und Standardabweichungen aus zwei $(Y x / s)$ bzw. drei Replikaten (CscA-Aktivität, $\mu$ )). 
$\csc B$ aus Escherichia coli W ein, die für eine Invertase und ein Transportprotein codieren [7]. Tatsächlich war P. putida damit in der Lage, auf Saccharose als alleiniger C-Quelle zu wachsen, allerdings mit einer deutlich niedrigeren Wachstumsrate $(\mu=0,27 \pm$ $0,01 \mathrm{~h}^{-1}$ ) als der Wildtyp auf den Monomeren Glucose und Fructose $\left(\mu=0,45 \pm 0,02 \mathrm{~h}^{-1}\right.$, [8]). Eine genauere Untersuchung des Mechanismus zeigte, dass ein gewisser Prozentsatz der Invertase CscA unspezifisch aus der Zelle entweicht und dort die Saccharose spaltet. Die damit verbundene transiente Akkumulation von Glucose und Fructose im Kulturmedium war offensichtlich ausreichend, um das Wachstum zu ermöglichen. In einem nächsten Schritt untersuchten wir die extra- und intrazelluläre Aktivität der Invertase CscA und konnten über 90 Prozent der Aktivität im intrazellulären Bereich nachweisen (Abb. 1A), was vermuten lässt, dass der Transport der Saccharose in die Zelle den limitierenden Schritt darstellt. Durch Versuche mit Zellen, die entweder nur CscA oder zusätzlich CscB produzierten, konnten wir zeigen, dass in P. putida die Anwesenheit von CscB keinen Vorteil bei Wachstum auf Saccharose brachte (Abb. 1B). Die Stämme unterschieden sich weder in der Wachstumsrate noch in der Biomasseausbeute, obwohl CscB nachweislich produziert wurde und in der Membran lokalisiert war [8]. Die Permease CscB schien also in diesem Stamm nicht funktional zu sein. In E. coli K12 hingegen waren beide Gene $\csc A$ und $\csc B$ notwendig (und ausreichend!) um ein gutes Wachstum auf Saccharose zu erreichen [7].

Warum funktionierte das nicht in gleichem Maße in P. putida? Im Allgemeinen müssen drei wichtige Aspekte in Betracht gezogen werden, wenn durch metabolic engineering ein neues Substrat einem Gram-negativen Organismus zugänglich gemacht werden soll:

(i) Das Substrat passiert zunächst die äußere Membran,

(ii) anschließend erfolgt ein Transport über die Cytoplasmamembran und

(iii) schlussendlich tritt es in den zellulären Stoffwechsel ein.

Die Permease CscB sollte den Transport über die Cytoplasmamembran ins Zellinnere ermöglichen, wo durch Spaltung der Saccharose durch CscA der Eintritt in den zelleigenen Stoffwechsel stattfinden sollte. Ein deutlicher Unterschied zwischen E. coli und $P$. putida zeigt sich jedoch in der strukturellen Zusammensetzung der äußeren Mem- bran. Im Gegensatz zu Enterobakterien finden sich in der äußeren Membran der Pseudomonaden keine unspezifischen Diffusionsporine wie OmpF und $\mathrm{OmpC}$, sondern primär spezifische Kanalproteine für die Aufnahme bestimmter Stoffe. Die Abwesenheit unspezifischer Porine spiegelt sich in einer deutlich reduzierten Permeabilität der äußeren Membran wider [9] und ist auch einer der Gründe für die intrinsische Resistenz dieser Organismen gegenüber einer Vielzahl von Umweltgiften und Antibiotika.

Identifikation eines natürlichen Stoffwechselwegs zum Saccharoseabbau in $P$. protegens Pf-5

Um die Saccharoseaufnahme in die Zelle von $P$. putida zu verbessern, gab es unterschiedliche Ansatzpunkte. Zum einen konnte nicht zweifelsfrei gezeigt werden, dass die Permease $\mathrm{CscB}$ aus E. coli $\mathrm{W}$ in P. putida funktional ist, zum anderen deutete vieles darauf hin, dass der Transport der Saccharose durch die äußere Membran gestört ist. Deshalb suchten wir in Genomen anderer Gram-negativer Organsimen nach putativen SaccharoseOperonen mit Potenzial für die Verbesserung der Saccharoseaufnahme in P. putida, sei es durch das Vorhandensein einer alternativen Permease oder eines geeigneten Porins.

Mit $\csc B$ von E. coli $\mathrm{W}$ als Matrize identifizierten wir durch Homologiesuche mittels BLAST ein Gencluster in P. protegens Pf-5, bestehend aus den offenen Leserahmen (ORF) PFL_3236 bis PFL_3239. Aufgrund von Homologie annotierten wir die ORFs als CSCR (Repressor, PFL_3236), CSCA (Saccharose-Hydrolase, PFL_3237), CSCB (Permease, PFL_3238) und CSCY (Porin, PFL_3239), zusammen bilden sie das CSCRABY-Operon. $P$. protegens Pf-5 gehört der Gattung Pseudomonas an und ist damit taxonomisch deutlich enger mit $P$. putida verwandt als $E$. coli.

\section{Das cscRABY-Operon ermöglicht zuverlässiges Wachstum auf Saccharose in $P$. putida}

An erster Stelle stand nun der Nachweis, ob dieses Operon tatsächlich das Wachstum auf Saccharose ermöglicht. Dazu konstruierten wir unterschiedliche Plasmide, die entweder das gesamte Operon (pSEVA221-CSCRABY) oder nur das für das Porin kodierende Gen CSCY (pSEVA434-CSCY) bzw. die Gene des Operons ohne das Porin (pSEVA221-CSCRAB) trugen, und uns somit erlaubten, die Rolle der einzelnen Komponenten zu untersuchen.

Nach Konjugation des Plasmids, welches das gesamte CscRABY-Operon trug, war $P$. putida in der Lage, auf Saccharose zu wachsen (Abb. 2). Wurden jedoch nur die Gene cscRAB eingebracht, konnte auch nach drei Tagen Inkubation noch kein Wachstum beobachtet werden. Durch Komplementation dieses Stamms mit dem Plasmid pSEVA434${ }_{C s C} Y$ wurde das Wachstum wiederhergestellt. Damit zeigten wir, dass CscY wichtig für die Aufnahme von Saccharose in die Zelle ist, indem es den Transport über die äußere

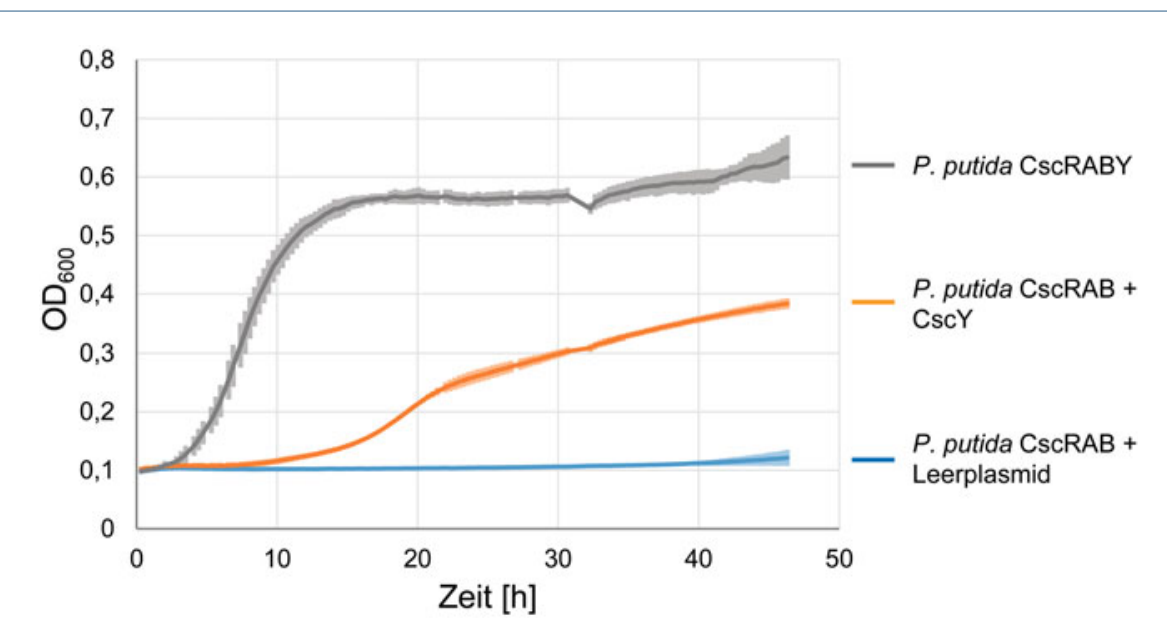

$\triangle$ Abb. 2: Wachstum von Pseudomonas putida (pSEVA221-cscRABY) (grau) und Komplementationsstudien mit $P$. putida (pSEVA221-CSCRAB, pSEVA434-cSCY) (orange) und $P$. putida (pSEVA221$\operatorname{cscRAB}$, pSEVA434) (blau) mit $3 \mathrm{~g} / \mathrm{L}$ Saccharose in M9-Medium im 96-Well-MikrotiterplattenLesegerät. Die Vorkulturen wurden in LB-Medium angezogen. Dargestellt sind die Mittelwerte und das 95 \%-Konfidenzintervall aus neun Replikaten (drei unabhängige Kulturen mit je drei technischen Replikaten). 

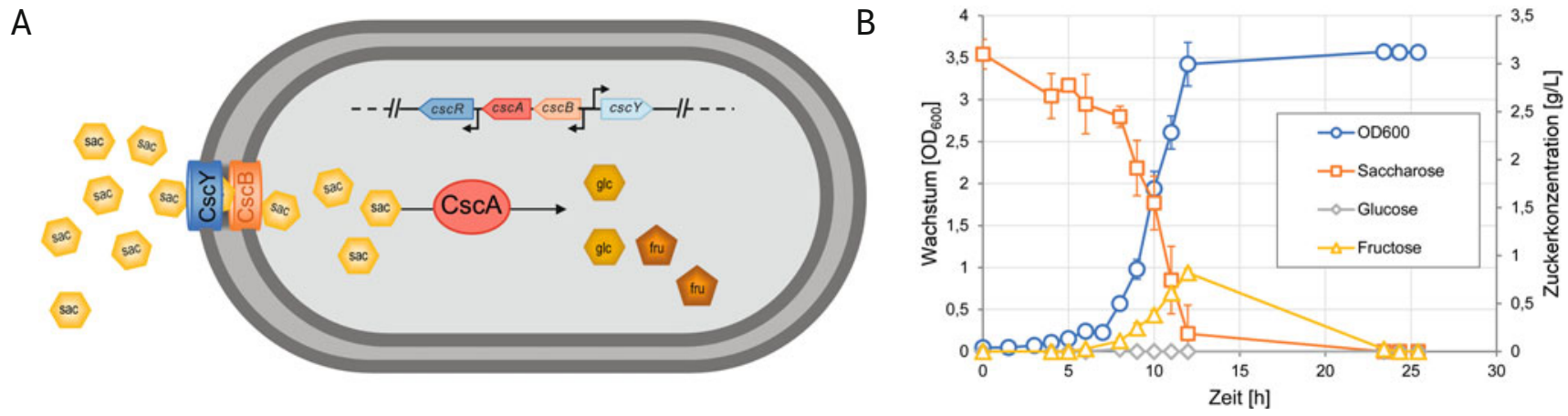

$\Delta$ Abb. 3: A, Überblick über den Mechanismus der Saccharoseaufnahme in Pseudomonas putida att::cscRABY. Saccharose (sac) wird durch das Porin CscY ins Periplasma und den Transporter CscB ins Cytoplasma transportiert. Dort wird sie durch die Aktivität der Invertase CscA in die Monomere Glucose (glc) und Fructose (fru) gepalten. B, Wachstum und Spaltung von Saccharose in P. putida att::cscRABY über die Zeit im Schüttelkolbenexperiment mit M9-Minimalmedium mit Saccharose (Mittelwerte und Standardabweichungen aus drei unabhängigen Replikaten).

Membran ermöglicht. Interessanterweise stellten wir fest, dass die Abhängigkeit des Wachstums von dem Porin CscY nur in den Kulturen beobachtet werden konnten, die aus einer Vorkultur angeimpft worden waren, welche auf LB-Medium kultiviert worden war. Wurde hingegen die Hauptkultur mit Zellen einer in Mineralmedium gezogenen Vorkultur inokuliert, wuchsen auch die Zellen ohne CscY auf Saccharose, und zwar mit durchaus vergleichbaren Raten. Dieses Phänomen liegt vermutlich in dem unterschiedlichen metabolischen Regime der Kulturen begründet. Zellen, die in Mineralmedium mit Glucose wachsen, zeigen glykolytische Aktivität, wohingegen LBgezogenen Zellen Gluconeogenese durchführen müssen. Diese unterschiedlichen Lebensarten führen auch zu Unterschieden in der äußeren Membran [10,11]. Vermutlich reicht das Set von Transportern und Porinen, welches bei Wachstum in Mineralmedium auf Glucose ausgebildet wird, aus, um in der Hauptkultur die Saccharose in das Innere der Zelle zu transportieren. Bei Wachstum der Vorkultur in LB-Medium scheint die Zusammensetzung anderer Art zu sein, sodass die Saccharose nicht in die Zelle gelangen kann. Mittels PeriplasmaSchwellungsexperimenten konnten wir die Permeabilität der äußeren Membran abschätzen und beobachten, dass in der Tat die Saccharose schneller durch die äußere Membran von Zellen, die in Mineralmedium mit Glucose angezogen worden waren, diffundieren konnte, als durch die von in LBMedium kultivierten Zellen [12].

\section{Genomintegration von cscRABY führt zu einem stabilen Wachstum auf Saccharose}

Als nächster Schritt wurde das $\operatorname{cscRABY-}$ Operon mittels des mini-Tn7-Transposons in das Genom von P. putida integriert, um einen stabilen Phänotyp zu erhalten und die Notwendigkeit von Antibiotikagabe zur Selektion des Plasmids zu umgehen (Abb. 3). Die nun erreichten Wachstumsraten waren vergleichbar zu denen auf den Monomeren Fructose und Glucose [12]. Im Vergleich zu $P$. putida $\csc A B$, welcher die Gene aus $E$. coli $\mathrm{W}$ integriert hatte, konnten wir die Raten um ungefähr 60 Prozent verbessern. Der postulierte Mechanismus der Saccharoseaufnahme, das Wachstum und die Saccharosespaltung über die Zeit sind in Abbildung $\mathbf{3}$ dargestellt. Nach dem Transport über die äußere Membran mittels CscY und die Cytoplasmamembran durch CscB, wird die Saccharose intrazellulär durch die Aktivität von CscA zu den Monomeren Glucose und Fructose gespalten (Abb. 3A). Glucose kann direkt durch die Glucokinase (Glk) verstoffwechselt werden, wohingegen Fructose erst wieder ausgeschleust werden muss, um dann über das Fructose-Phosphotransferasesystem (PTS ${ }^{\mathrm{Fru}}$ ) aufgenommen und somit dem Stoffwechsel von $P$. putida zugänglich gemacht zu werden. P. putida besitzt keine funktionale Fructokinase, die eine direkte Einschleusung der Fructose in den Metabolismus ermöglichen würde [13]. Dies erklärt die transiente Akkumulation von Fructose im Medium (Abb. 3B).

\section{Ausblick}

P. putida ist ein interessanter Organismus für biotechnologische Anwendungen, da er ein robustes metabolisches und biochemisches Umfeld bereitstellt, das die Implementierung einer Vielzahl verschiedener Vorgänge ermöglicht. Hier zeigten wir, wie mittels rationalem Design das Substratspektrum von $P$. putida um einen weiteren Zucker erweitert werden konnte. Darüber hinaus demonstriert diese Arbeit, dass die äußere Membran eine kritische Barriere bei der Implementierung neuer Stoffwechselwege sein kann, die Beachtung im Design von genetischen Elementen und Produktionsstämmen zu finden hat. Porinen wird in der Regel beim metabolic engineering nicht allzu viel Aufmerksamkeit geschenkt. Abhängig von dem zu transportierenden Stoff können sie aber von essenzieller Bedeutung sein, um einen stabilen Phänotyp zu erhalten. Wenn bei der biotechnologischen Produktion die Aufnahme des Stoffs in die Zelle den limitierenden Schritt darstellt, kann durch die Verwendung eines geeigneten Porins die Produktivität gesteigert werden. In anderen Fällen kann ein Porin die allgemeine Fitness erhöhen, da es den Transport eines ansonsten giftigen Stoffs über die äußere Membran bewerkstelligt. Hier war das Porin CscY entscheidend, um einen stabilen Saccharose-verstoffwechselnden Phänotyp zu erhalten.

\section{Danksagung}

Unser Dank gilt Prof. Kirsten Jung (LMU, München) für die Überlassung von Pseudomonas protegens Pf-5 und Víctor de Lorenzo (CNB, Madrid) für die Plasmide aus der SEVA Bibliothek. Ein besonderer Dank geht an Karina Hobmeier, Lukas Schmauder und Peter Sinner, die an der Bearbeitung dieses Themas beteiligt waren.

\section{Literatur}

[1] Nikel PI, Chavarría M, Danchin A, de Lorenzo V (2016) From dirt to industrial applications: Pseudomonas putida as a synthetic biology chassis for hosting harsh biochemical reactions. Curr Opin Chem Biol 34: 20-29

[2] Poblete-Castro I, de Acuña JMB, Nikel PI et al. (2017) Host organism: Pseudomonas putida. In: Wittmann C, Liao JC (Hrsg.) Industrial biotechnology: Microorganisms. Volume 1, Wiley-VCH Verlag, New York, 299-326

[3] Nikel PI, de Lorenzo V (2018) Pseudomonas putida as a functional chassis for industrial biocatalysis: from native biochemistry to trans-metabolism. Metab Eng 50: 142-155 
[4] Nikel PI, Martínez-García E, de Lorenzo V (2014) Biotechnological domestication of pseudomonads using synthetic biology. Nat Rev Microbiol 12: 368-379 [5] Nikel PI, Chavarría M, Führer T et al. (2015) Pseudomonas putida KT2440 strain metabolizes glucose through a cycle formed by enzymes of the Entner-Doudoroff, Embden-Meyerhof-Parnas, and pentose phosphate pathways J Biol Chem 290: 25920-25932

[6] Nikel PI, Fuhrer T, Chavarría M et al. (2021) Reconfiguration of metabolic fluxes in Pseudomonas putida as a response to sub-lethal oxidative stress. Isme J, doi: 10.1038/s41396020-00884-9

[7] Sabri S, Nielsen LK, Vickers CE (2012) Molecular control of sucrose utilization in Escherichia coli W, an efficient sucrose-utilizing strain. Appl Environ Microbiol 79: 478-487 [8] Löwe H, Schmauder L, Hobmeier K et al. (2017) Metabolic engineering to expand the substrate spectrum of Pseudomonas putida toward sucrose. MicrobiologyOpen 6: $\mathrm{e} 00473$

[9] Hancock REW, Brinkman FSL (2002) Function of Pseudomonas porins in uptake and efflux. Annu Rey Microbiol 56: 17-38

[10] Thompson DK, Chourey K, Wickham GS et al. (2010) Proteomics reveals a core molecular response of

Pseudomonas putida $\mathrm{F} 1$ to acute chromate challenge. BMC Genomics 11: 311
[11] Choi C-W, Park EC, Yun SH et al. (2014) Proteomic characterization of the outer membrane vesicle of Pseudomonas putida KT2440. J Proteome Res 13: 4298-4309 [12] Löwe H, Sinner P, Kremling A, Pflüger-Grau K (2018) Engineering sucrose metabolism in Pseudomonas putida highlights the importance of porins. Microb Biotechnol 13: 97-106

[13] Sawyer MH, Baumann P, Baumann L et al. (1977) Pathways of D-fructose catabolism in species of Pseudomonas. Arch Microbiol 112: 49-55

Funding note: Open Access funding enabled and organized by Projekt DEAL. Open Access: Dieser Artikel wird unter der Creative Commons Opention Vervielfältigung, Bearbeitung, Verbreitung und Wiedergabe in jeglichem Medium und Format erlaubt, sofern Sie den/die ursprünglichen Autor(en) und die Quelle ordnungsgemäß nennen, einen Link zur Creative Commons Lizenz beifugen und angeben, ob Änderungen vorgenommen wurden. Die in diesem Artikel enthaltenen Bilder und sonstiges Drittmaterial unterliegen ebenfalls der genannten Creative Commons Lizenz, sofern sich aus der Abbildungslegende ichts anderes ergibt. Sofern das betreffende Material nicht unter der Wertich Weterverwend Rechteinhabers einzuholen. Weitere Details zur Lizenz entnehmen Sie bitte der izenzinformation auf http://creativecommons.org/licenses/by/4.0/deed. de.

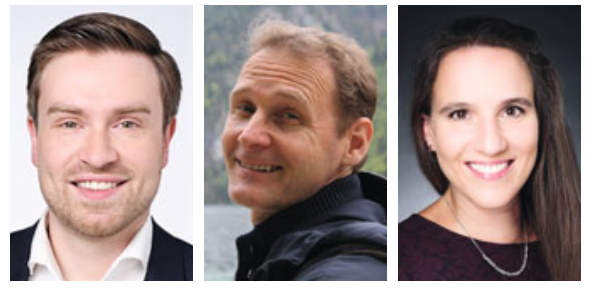

Hannes Löwe, Andreas Kremling und Katharina Pflüger-Grau (v. I. n. r.)

\section{Korrespondenzadresse:}

Dr. Katharina Pflüger-Grau

Professur für Systembiotechnologie

Technische Universität München (TUM)

Boltzmannstraße 15

D-85748 Garching

k.pflueger-grau@tum.de

\section{Hier steht} eine Anzeige. 